\title{
ESTUDIO PILOTO COMPARATIVO DE SALUD ORAL RELACIONADA A LA CALIDAD DE VIDA EN ESCOLARES DEL CANTÓN CAÑAR ECUADOR 2015
}

\author{
ENCALADA-VERDUGO LILIANA* \\ GARCIA RUPAYA CARMEN ${ }^{* *}$ \\ DEL CASTILLO LOPEZ CÉSAR ${ }^{* * *}$
}

Recibido: 06/09/2015

Aceptado: 13/10/2015

\section{RESUMEN}

Se realizó un estudio piloto comparativo con el objetivo de determinar el impacto de las condiciones orales en la calidad de vida de los escolares del sector urbano y rural del Cantón Cañar, Ecuador a través de la aplicación del cuestionario Child OIDP, en el año 2015. Se realizó un Estudio trasversal observacional mismo que evaluó a 37 niños de 11 y 12 años de edad, de dos escuelas del Cantón Cañar que cumplieron con los criterios de selección, las escuelas intervenidas fueron en zona urbana (Luis Roberto Chacón) y rural (Huayrapungo). Los datos fueron recolectados a través de una entrevista personal con cada niño empleando el instrumento Child OIDP versión peruana. Según los resultados obtenidos los principales desempeños afectados fueron el sonreír en un $60 \%$, comer en un $57.1 \%$, socializar en un $54.3 \%$, limpiarse la boca en un $34.3 \%$; los menos afectados fueron erupción diente permanente en un $11.4 \%$, dormir en un $5.7 \%$, estudiar en un $2.9 \%$ y no se vio afectado el desempeño de hablar. En conclusión, tanto los niños de la escuela urbana y rural presentaban al menos un impacto que afectaba a un desempeño; siendo el desempeño sonreír el más afectado en la escuela urbana y el desempeño comer en la escuela rural.

PALABRAS CLAVE: Calidad de vida, salud oral, población urbana, población rural.

\begin{abstract}
A comparative pilot study was carried out with the objective of determining the impact of oral conditions on the quality of life of school children both from rural as well as urban sectors in Cañar Canton, Ecuador, through the application of OIDP Child questionnaire in 2015. An observational cross-sectional study was conducted which evaluated 37 children between 11 and 12 years old from two schools in Cañar that met the selection criteria. The schools involved were Luis Roberto Chacón (urban) and Huayrapungo (rural). Data were collected through a personal interview with each child using the OIDP, peruvian versión. According to the results obtained the main performances affected were: smiling in $60 \%$, eating in $57.1 \%$, socializing in $54.3 \%$, and cleaning their mouth in $34.3 \%$. The least affected actions were: eruption of a permanent tooth in $11.4 \%$, sleeping in $5.7 \%$ and studying in $2.9 \%$. The speaking function was not affected. In conclusion, both urban and rural school children presented at least one impact that affected their performance, being smiling the one most affected at the urban school and eating at he rural one.
\end{abstract}

KEY WORDS: Quality of life, oral health, urban population, rural population

\section{INTRODUCCIÓN}

La caries dental a nivel del mundo, según la Organización Mundial de la Salud (OMS), ocupa uno de los primeros lugares en afecciones bucodentales de los niños, Ecuador no es la excepción, la prevalencia según los reportes del Ministerio de Salud Pública (MSP), demuestran que esta patología ocupa el primer lugar de afecciones bucodentales ${ }^{1}$.

Existen otras enfermedades bucodentales que afectan a la niñez ecuatoriana, tales como gingivitis y maloclusión, que son clasificadas en una descripción meramente clínica, siendo la caries la de mayor prevalencia y la que se puede cuantificar mediante índices.

La definición compleja de salud, con enfoque multidisciplinario, aplicado a la integridad del ser humano, obliga a relacionar la salud bucodental y su deterioro con las condiciones sociales, culturales y económicas de los sectores urbanos y rurales, e identificar cómo afecta en el aspecto emocional de las personas. ${ }^{1}$

La calidad de vida es un complemento del diagnóstico clínico odontológico, cualquier patología buco dental puede estar afectando el desenvolvimiento normal de ese individuo. La calidad de vida con relación a la salud refiere la capacidad de adaptación del ser humano a la enfermedad. ${ }^{2}$

La calidad de vida en la población ecuatoriana está planteada en la Constitución de la República como "Sumak Kawsay" o "Buen Vivir, que se entiende como la interacción del hombre con la naturaleza, la interculturalidad y el respeto a la vida en torno a todas las funciones del cuerpo humano. El propósito de este estudio fue determinar cómo afectan las condiciones orales en la calidad de vida de los escolares según el sexo y la ubicación.

* Docente Maestrante en Estomatología, Universidad Católica de Cuenca.Ecuador

** Magister en Estomatología de la Universidad Católica Peruana Cayetano Heredia.Perú

*** Magister en Estomatología. Coordinador de Odontología Social de la Universidad Peruana Cayetano Heredia.Perú 


\section{MATERIALES Y MÉTODOS}

El estudio fue de tipo descriptivo observacional y transversal. La población estuvo conformada por 90 escolares de 11 y 12 años de edad que estuvieron matriculados en el periodo académico 2014-2015 en las Instituciones Educativas Luis Roberto Chacón y Unidad Educativa Huayrapungo, del Cantón Cañar. Al ser un estudio piloto la muestra fue obtenida basándonos en el criterio estadístico de que se requiere un mínimo de 30 para aplicar cálculo matemático; por ello se contó con 17 escolares urbanos y 18 escolares rurales sumando un total de 35 escolares. Se consideraron como criterios de inclusión los siguientes: escolares de 11 y 12 años de edad cumplidos, aparentemente sanos, de ambos sexos, con autorización de las instituciones educativas, con Consentimiento Informado de los padres y Asentimiento Informado de los escolares.

Los criterios de exclusión fueron: escolares con discapacidad psicológica no corregida. Se empleó el método de encuesta utilizando el cuestionario validado Child OIDP versión en español - Perú4.

No se ha hecho una validación de este cuestionario en Ecuador pero se recalca que la terminología es similar entre estos dos países, previo a la calibración recibida por Del Castillo, autor de uno los estudios previos, se aplicó la encuesta, en forma de entrevista en un ambiente aislado dentro de la institución educativa, en donde se tenía Cara a cara a cada niño, la duración de la entrevista fue entre 6 a 8 minutos por escolar, realizándose ocho entrevistas cada hora en el horario de mañana.

Se inició con la primera parte del instrumento en donde al escolar se le pregunta si en los últimos tres meses ha presentado uno de los 17 impactos que constan en el cuestionario y que haya sido un problema para el escolar, se marcó de color diferente cuando existió más de un impacto, luego se le pregunta al escolar por cada uno de los impactos si imposibilitó alguno de los ocho desempeños, si el escolar manifiesta que no existe desempeño afectado la severidad y frecuencia tienen el valor de cero, mientras que si manifiesta existencia de un desempeño afectado se procedió a determinar la severidad en escala de $1=$ poco, $2=$ regular, $3=$ severo, para esto se preguntó al escolar el impacto que presentó cuánto afecto el desempeño se les mostró unas caritas en forma de semáforo en donde el verde es 1,el amarillo es 2 y el rojo es 3 y el niño muestra cuanto afecto. Para determinar la intensidad fue necesario determinar la frecuencia de la dificultad en el desempeño.

Para valorar la frecuencia, en la escala de 1 a 3, se preguntó al escolar cuántas veces se presentó la dificultad en los últimos tres meses si fue de 1 a 2 veces el valor será 1 , si fue de 2 a 3 veces el valor será 2 y si fue más de 3 veces el valor es 3 . Se obtuvo la aprobación del Comité Institucional de Ética de la Unidad Académica de Ciencia Odontológica de La Universidad Católica de Cuenca, la autorización de los Directores de la Instituciones Educativas, el consentimiento de los padres de familia y el asentimiento de los niños.

\section{RESULTADOS}

Del total de escolares, se identificaron 26 mujeres $(71,4 \%)$ y 9 varones $(28,6 \%)$. En cuanto a la ubicación se encontró 17 escolares urbanos (48,5\%) y 18 escolares rurales $(51,5 \%)$

Tabla 1. Prevalencia del impacto de las condiciones orales sobre los desempeños diarios que afectan la calidad de vida en escolares de 11 y 12 años del Cantón Cañar Ecuador 2015.

\begin{tabular}{ccccc}
\hline $\begin{array}{c}\text { Actividades } \\
\text { diarias }\end{array}$ & \multicolumn{2}{c}{$\begin{array}{c}\text { Con Impacto } \\
\text { (Child-OIDP>0) }\end{array}$} & \multicolumn{2}{c}{$\begin{array}{c}\text { Sin Impacto } \\
\text { (Child-OIDP=0) }\end{array}$} \\
\hline Comer & 20 & 57,1 & 15 & 42,9 \\
Hablar & 0 & 0,0 & 35 & 100,0 \\
Limpiarse la & 12 & 34,3 & 23 & 65,7 \\
boca & 2 & 5,7 & 33 & 94,3 \\
Dormir & 4 & 11,4 & 31 & 88,6 \\
Emoción & 21 & 60,0 & 14 & 40,0 \\
Sonreir & 1 & 2,9 & 34 & 97,1 \\
Estudiar & 19 & 54,3 & 16 & 45,7 \\
Socializar & & & &
\end{tabular}


Tabla 2. Prevalencia total de los Impactos orales percibidos por los escolares del sector Urbano y Rural del Cantón Cañar Ecuador 2015.

\begin{tabular}{lcc}
\hline \multicolumn{1}{c}{ Condiciones Orales } & \multicolumn{2}{c}{ Prevalencia } \\
\hline Dolor de muela & $\mathrm{n}$ & $\%$ \\
Diente sensible & 19 & 17,14 \\
Diente cariado & 12 & 54,28 \\
Caida de un diente de leche & 2 & 34,28 \\
Dientes separados & 1 & 5,71 \\
Fractura de diente permanente & 1 & 2,85 \\
Color de los dientes & 17 & 2,85 \\
Forma o tamaño de los dientes & 3 & 48,57 \\
Posición de los dientes & 8 & 8,57 \\
Sangrado de encias & 10 & 22,85 \\
Inflamación de las encias & 0 & 0,0 \\
Sarro o Cálculo Dental & 0 & 0,0 \\
Llagas o heridas en la boca & 1 & 2,85 \\
Mal aliento & 17 & 48,57 \\
Deformación de la boca & 0 & 0,0 \\
Erupción de diente permanente & 4 & 11,42 \\
Pérdida de diente permanente & 1 & 2,85 \\
Otros & 0 & 0,0 \\
\hline
\end{tabular}

Tabla 3. Prevalencia de los Impactos orales percibidos por los escolares del sector Urbano y Rural del Cantón Cañar Ecuador 2015

\begin{tabular}{lcccc}
\hline \multicolumn{1}{c}{ Condiciones Orales } & \multicolumn{2}{c}{$\begin{array}{c}\text { Urbana } \\
(\mathrm{n}=17)\end{array}$} & \multicolumn{2}{c}{ Rural } \\
$(\mathrm{n}=18)$
\end{tabular}


Tabla 4. Prevalencia de los Impactos orales percibidos por los escolares según el sexo del sector Urbano y Rural del Cantón Cañar Ecuador 2015, según el sexo.

\begin{tabular}{lccccc}
\hline & \multicolumn{2}{c}{$\begin{array}{l}\text { Mujeres } \\
\text { Condiciones Orales }\end{array}$} & \multicolumn{2}{c}{$\begin{array}{c}\text { Varones } \\
\text { (n=26) }\end{array}$} & $\mathbf{X}^{2}$ \\
& $\mathbf{n}$ & $\mathbf{\%}$ & $\mathbf{n}$ & $\mathbf{\%}$ & $\mathbf{p}$ \\
\hline Dolor de muela & 5 & 19,23 & 1 & 11,11 & 0,01 \\
Diente sensible & 15 & 57,69 & 4 & 44,44 & 0.04 \\
Diente cariado & 10 & 38,46 & 2 & 22,22 & 0.06 \\
Caida de un diente de leche & 2 & 7,69 & 0 & 0,0 & 1,00 \\
Dientes separados & 0 & 0 & 0 & 0 & - \\
Fractura de diente permanente & 0 & 0 & 0 & 0,0 & - \\
Color de los dientes & 17 & 65,38 & 0 & 0 & - \\
Forma o tamaño de los dientes & 2 & 7,69 & 1 & 11,11 & 0,01 \\
Posición de los dientes & 4 & 15,38 & 4 & 44,44 & 0.04 \\
Sangrado de encias & 0 & 0 & 5 & 55,55 & 0,05 \\
Inflamación de las encias & 0 & 0 & 0 & 0 & - \\
Sarro o Cálculo Dental & 0 & 0 & 0 & 0 & - \\
Llagas o heridas en la boca & 1 & 3,84 & 0 & 0 & - \\
Mal aliento & 14 & 53,84 & 3 & 33,33 & 0,03 \\
Deformación de la boca & 0 & 0 & 0 & 0 & - \\
Erupción de diente permanente & 3 & 11,53 & 1 & 11,11 & 0,01 \\
Pérdida de diente permanente & 0 & 0 & 1 & 11,11 & 0,01 \\
\hline
\end{tabular}

\section{DISCUSIÓN}

El presente estudio piloto se realizó en niños de 11 y 12 años de edad de la escuela urbana "Luis Roberto Chacón" y de la escuela rural "Huayrapungo" del Cantón Cañar. Los resultados nos permiten enunciar que los desempeños afectados fueron el sonreír en un $60 \%$, comer en un $57.1 \%$, socializar en un $54.3 \%$, limpiarse la boca $34.3 \%$; los menos afectados fueron emoción $11.4 \%$, dormir en un $5.7 \%$, estudiar en un $2.9 \%$ y no se vio afectado el desempeño de hablar, resultados también encontrados por Gherumpong ${ }^{3}$ Sanchez $^{4}$ y Bernabé ${ }^{5}$.

De acuerdo al sexo los impactos más afectados al sexo femenino fueron color de dientes, mal aliento y dientes sensibles, mientras que al sexo masculino fue el sangrado de encías, estos hallazgos coinciden con los de Olivera Sheiham. Los impactos orales que fueron más percibidos por los escolares del sector urbano del Cantón cañar fueron: el color de los dientes en un $100 \%$, diente sensible en $70.58 \%$ y mal aliento en un $58.82 \%$; los menos afectados con porcentajes inferiores al $10 \%$ fueron dolor de muela, caída de diente de leche, dientes separados, fractura de dientes, tamaños de los dientes, sangrado de encías y heridas; los impactos orales no afectados fueron: inflamación de las encías, sarro, deformación de la boca y perdida de dientes permanentes. Mientras que en el sector rural los impactos más afectados fueron: sangrado de encías en un $52,94 \%$, diente cariado $47.05 \%$, diente sensible $41,17 \%$ y dolor de muela con $23.52 \%$; los impactos menos afectados con un porcentaje inferior al $10 \%$ tenemos forma y tamaño de dientes, erupción de dientes permanentes y perdida de dientes definitivos; los impactos no afectados fueron: caída de diente de leche, dientes separados, fractura de diente permanente, color de los dientes, inflamación de encías, sarro, heridas y deformación de la boca. 


\section{CONCLUSIONES}

Los desempeños comer y socializar fueron los más afectados tanto en escolares del sector urbano como del sector rural del Cantón Cañar.

Los escolares del sector urbano tuvieron un $100 \%$ de afección en el desempeño sonreír, mientras que los escolares del sector rural apenas se vieron afectados en el desempeño sonreír en un $23 \%$.

Se puede concluir que la parte estética que involucra el desempeño sonreír afecta más a la niñez del sector urbano que a la del sector rural, los demás desempeños están afectados en porcentajes similares en las dos zonas.

\section{REFERENCIAS BIBLIOGRÁFICAS}

1. Moreno, G. La definición de salud de la Organización Mundial de la Salud y la interdisciplinariedad. Sapiens. Revista Universitaria de Investigación. 2008; 9(1), 93.

2. Schwartzmann L. Calidad de vida relacionada con la salud: aspectos conceptuales. Cienc enferm. 2003; 9(2), 09-21.

3. Gherunpong S, Tsakos G, Sheiman A. A sociodental approach to assessing dental needs of children: concept and models. Int J Paediatr Dent. 2006; 16:81-88.

4. Sánchez P. Impacto de las condiciones orales sobre la calidad de vida en escolares de 11 y 12 años de edad de Zapallal-Puente Piedra .Lima. 2007; 1:36.

5. Bernabé E, Flores-Mir C, Sheiman A. Prevalence, intensity and extent of Oral Impacts on Daily Performances associated with self-perceived malocclusion in 11-12-year old children. BMC Oral Health. 2007;7(6):1-7.

6. Tsakos G, Gherunpong S, Sheiham A. Can Oral Health-Related Quality of Life Measures Substitute for Normative Needs Assessments in 11 to 12-year-old Children? J Public Health Dent. 2006;66(4)2638.

7. World Health Organization. Oral health surveys: basic methods. World Health Organization; $1986 ; 4: 3$.

8. Locker D. Measuring oral health: a conceptual framework. Community Dent Health. 1988; 5:3-18.

9. Shamrany M. Oral health-related quality of life: A broader perspective. Eastern Mediterranean Health Journal (EMHJ). 2006; 12(6): 894-901.

10. Sischo L, Broder H. Oral Health-related Quality of life: What, Why, How, and Future Implications. J Dent Res. 2011; 90(11):1264-70.

11. Castro R, Portela M, Leao A, VasconcellosM.Oral Health-related quality of life of 11-12-year-old public school children in Rio de Janeiro. Community Dent Oral Epidemiol. 2011; 39:36.

12. Bernabé E, Tsakos G, Sheiman A. Intensity and extent of Oral Impacts on Daily Performances by type of self-perceived oral problems. Eur J Oral Sci. 2007; 115:111-6.

13. Esteve M, Roca J. Calidad de vida relacionada con la salud: un nuevo parámetro a tener en cuenta. MedClin 1997; 108:458-9.

14. Gherunpong S, Tsakos G, Sheiman A. The prevalence and severity of oral impacts on daily performances in Thai primary school children. Health Qual Life outcomes. 2004; 2:17.

15. Walter M, Woronuk J. Determinants of oral health-related quality of life in a cross-cultural GermanCanadian sample. J Public health. 2007; 15.43-50.

16. Velarde-Jurado E, Avila-Figueroa C. Evaluación de la calidad de vida. Salud Pública Mex 2002; 44(4):349-61.

17. Calidad de vida relacionada con la salud (CVRS) en la infancia y la adolescencia: revisión bibliográfica y de los instrumentos adaptados en España. GacSanit 2001.15:34-43.

18. Donovan K, Sanson-Fisher R, Redman S. Measuring quality of life in cancer patients. J ClinOncol 1989; 959-68.

19. Jokovic A, Locker D, Stephens M, Kenny D, Tompson B, Guyatt G. Validity and reliability of a questionnaire for measuring child oral-health-related quality of life. J Dent Res. 2002;81: 459-63.

20. Filstrup SL, Briskie D, Da Fonseca M, Lawrence L, Wandera A, Inglehart, M. Early childhood caries and quality of life: child and parent perspectives. Pediatr Dent. 2003; 25:431-40.

21. Gherunpong S, Tsakos G, Sheiman A. Developing and evaluating an oral health-related quality of life index for children; the Child-OIDP. Community Dent Health. 2004; 21:161-9.

22. Paredes-Martínez, E. Díaz-Pizán, M. E. Impacto de las condiciones bucales sobre la calidad de vida en escolares del Distrito de San Juan de Miraflores. Lima, Perú. REH. 2014; 24(3), 171.

23. Del Castillo C. Impacto de las condiciones orales sobre la calidad de vida relacionada a la salud (CVRS) de escolares del ámbito rural de Piura en el año. 2010; 9:3.

24. De Olivera CM, Sheiham A. The relationship between normative orthodontic treatment need and oral health-related quality of life. Community Dent Oral Epidemiol 2003; 31: 426-36. 